\title{
THE INFLUENCE OF BUOYANCY ON THE OSCILLATORY THERMOCAPILLARY CONVECTION WITH SMALL BOND NUMBER $\dagger$
}

\author{
WEN-RUI HU \\ Institute of Mechanics, Academia Sinica, Beijing, China
}

(Received 15 October 1988)

\begin{abstract}
The mechanism of the onset of oscillatory thermocapillary convection is analysed for a liquid bridge with small Bond number. It shows that the buoyant forces are significant in the internal region, where the direction of local temperature gradient may turn to the direction of gravity in the case of larger Marangoni number, and the oscillatory state will be excited if the instability condition of convection driven by the buoyant force is satisfied, although the thermocapillary convection is primarily driven by the gradient of surface tension. Therefore, the critical state for the onset of the oscillation is probably dependent on several typical parameters such as the Marangoni number. Grashof number, Prandtl number and Bond number, but not by the only Marangoni number. A simplified model is suggested for a silicon oil bridge. The theoretical values of critical Marangoni numbers with the results of experiments on the ground, in the rocket, and the Spacelab on-board the Shuttle. That is, the critical Marangoni number will be larger for the case of smaller Bond number such as the experiments on board the Shuttle. This result is of great benefit to space material processes for the delay of oscillatory state appearance.
\end{abstract}

\section{INTRODUCTION}

The convection driven by the non-uniform surface tension at the free surface or inter-surface has been studied since Marangoni researched the non-uniform surface tension coming from the gradient of concentration in 1871[1]. Then, the convection of a liquid film and other interesting engineering problems stimulated the research of Marangoni convection, which is driven by the non-uniform surface tension produced by either gradient of temperature or concentration or electrical potential[2,3]. There are many papers dealing with the flow mechanism from the view point of fluid mechanics, e.g. the convection in a liquid layer studied by Pearson[4] and others $[5,6]$.

The microgravity fluid mechanics gradually becomes the nuclear part of microgravity sciences[7]; it lays a foundation for understanding the space materials process. In the space process of crystal growth in floating region, certain quantity of mass transportation are needed on the one hand, and unfavourable disturbations produced by convections should be avoided on the other hand. Therefore, a lot of research of numerical simulations and laboratory experiments, in addition to a few analytical works, have been completed for the models of liquid bridge. The pioneering works suggested by Chang and Wilcox for numerical simulation of thermocapillary convection[8], Ostrach for thoeoretical research[9] and experiment in drop tower[10], and Napolitano for the review[11].

+Paper IAF-88-365 presented at the 39th Congress of the International Astronautical Federation, Bangalore, India, 8-15 October 1988.
In the present paper, a mechanism is suggested for explanation of the oscillatory thermocapillary convection, which may be produced by the instability of buoyant forces. The temperature gradient in local regions may be parallel to the direction of gravity if the applied temperature difference is large enough. In this case, the buoyancy instability could be induced and developed. In Section 2, the physical model is discussed, and the influence of the buoyant force is analysed in Section 3. The comparison of critical Marangoni numbers between the calculated values and the experimental values are given in Section 4, and the results show in agreement.

\section{PHYSICAL MODEL}

A liquid bridge, as shown in Fig. 1 was discussed and is confined by two coaxial circular solid rods of

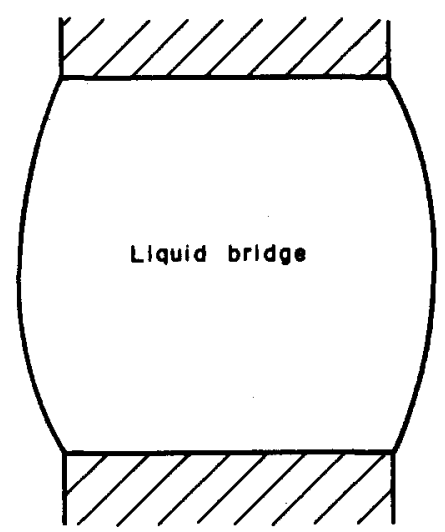

Fig. 1. Liquid bridge model scheme. 
same diameters $d$ and height $l$. The temperatures are $T_{0}$ at the colder lower rod and $T_{0}+\Delta T$ at the warm upper rod, and the experimental liquid floats between the rods with a free surface, where the surface tension $\sigma(T)$ is not uniform, because the temperature is not uniform at the surface of the liquid bridge produced by the temperature difference between the upper and lower rods. The gradient of surface tension drives the convection in the liquid, and the convection is coupled with the thermodynamical processes. It is believed that the buoyant force is relatively unimportant in comparison with the dominant effect of thermocapillary flow in the microgravity experimental environments such as in the rocket or on-board the Space Shuttle. In the ground laboratory, the thermocapillary effect could be dominated relative to the buoyancy if the typical scale of the experimental sample is reduced. The Bond number is defined as the ratio of buoyancy and surface tension gradient, small Bond number implies that the effect of buoyant force is less important than that of surface tension. In this case, the microgravity experiment could be simulated on the ground.

In the discussion of the free convection problem which is dominated by the buoyancy, two main dynamical simulation parameters are introduced, the Grashof number $\mathrm{Gr}=g \beta \delta T L^{3} / v^{2}$ and the Prandtl number $\operatorname{Pr}=v / \kappa$, where $\beta, v$ and $\kappa$ are, respectively, the thermal expansion coefficient, the kinematic viscosity coefficient and thermal diffusion coefficient, $L$ the typical scale and $\delta T$ the temperature difference whose gradient is parallel to the direction of gravity. In the stratified flow problem, we use the Rayleigh number $\mathbf{R a}=\mathbf{G r} \cdot \mathbf{P r}$, which is a parameter to measure the overall influences between buoyancy, viscosity and thermal transfer. For the problem of thermocapillary flow, the Marangoni number is defined as

$$
\mathrm{Ma}=-\frac{\frac{\mathrm{d} \sigma}{\mathrm{d} T} \Delta T L}{v \kappa}
$$

It is a parameter to measure the overall effects between the gradient of surface tension, viscosity and thermal transfer, and is similar to the implication of the Rayleigh number in the problem dominated by the buoyant force. There are many ground experiments of thermocapillary flow with small Bond number[12-16]. The temperature distribution at the surface of a liquid bridge changes with the value of Marangoni number: it is nearly linear if $\mathrm{Ma}$ is smaller; it appears as an S-shape and associates a single cell separated into several cells in the liquid bridge if $\mathrm{Ma}$ is increased; it becomes oscallatory and the flow pattern varies periodically as a spatial and temporal process if $\mathrm{Ma}$ is over a critical value $(\mathrm{Ma})_{\mathrm{c}}$. Similar results have been obtained in the large-scale experiments of thermocapillary convection in the rocket flight and on-board the Space Shuttle, but the critical Marangoni numbers appear obviously to have different values[17]. The value of $(\mathrm{Ma})_{c}$ is nearly $2 \times 10^{4}$ for the small liquid bridge on the ground, (1-2) $\times 10^{5}$ for the European rocket experiments of TEXUS program, and no less than $4 \times 10^{5}$ for the Spacelab experiment on-board the Space Shuttle. The appearance of an oscillary state will seriously impair the quality of materials produced by the space process, therefore, the mechanism of oscillation and the value of $(\mathrm{Ma})_{\mathrm{c}}$ are of deep concern. Monti has summarized the experimental results in a better approach by substituting the Weber number for Marangoni number[17]. In the present paper, we want to explain the reason for having different experimental values of $(\mathrm{Ma})_{\mathrm{c}}$ by the approach of mechanism analysis.

\section{THE INFLUENCE OF BUOYANCY}

Both the thermocapillary convection experiments on ground and in space satisfy the condition of the small Bond number: the influence of buoyancy is present as a Bond number and is not zero. Schwabe et $a l$. reduced the velocity by two orders of magnitude by surface treatment for reducing the driving force of surface tension. This result shows that the buoyant force is secondary in driving the fluid convection. However, their results show also that the buoyant force is important in the thermodynamical processes.

(a)

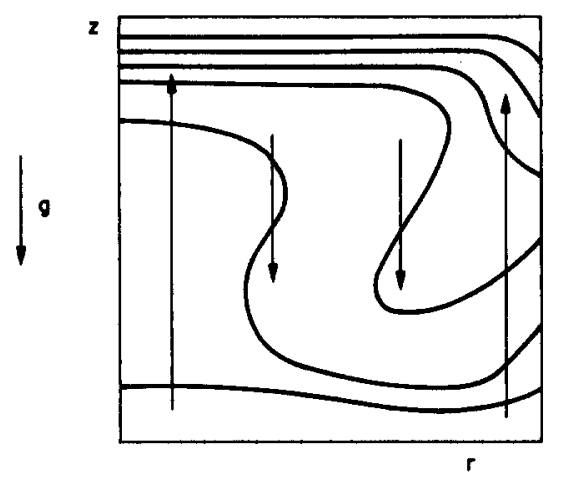

(b)

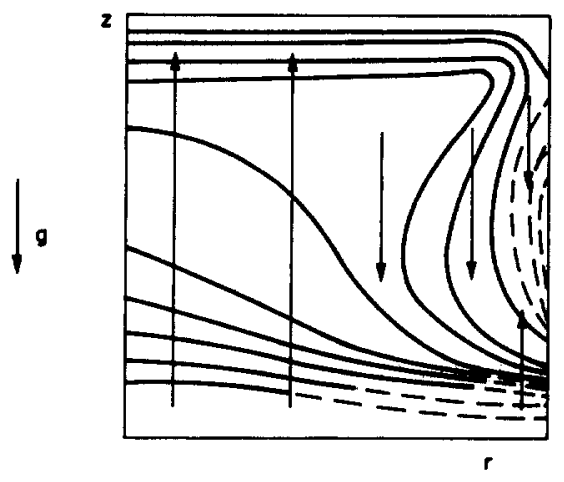

Fig. 2. The distributions of isotherms in the liquid bridge when $\Delta T$, or $\mathrm{Ma}$, is relatively larger, the arrows indicate the direction of the temperature gradient. 
In the liquid bridge experiments, the upper rod is usually warmer than the lower rod by a temperature difference $\Delta T$; that state is stable because the direction of temperature gradient is opposite to the gravitational direction. However, the reversed temperature gradient $\delta T$ appears in the local region of a liquid bridge if more heat is needed to transfer from the rod into the liquid as $\Delta T$ is increased. In this case, the temperature gradient is parallel to the gravity in local region as shown in Fig. 2 for the typical isotherms of different typical Ma. The reversed temperature difference $\delta T$ may increase as the increasing applied temperature difference $\Delta T$, and it associates an S-shape temperature distribution at the surface as shown in Fig. 2(b). Therefore, a local region with parellel directions between temperature gradient and gravity may appear in the liquid bridge if the applied temperature difference $\Delta T$ or $\mathrm{Ma}$ is increasing. The influence of buoyant forces will be important in this local region, and will strengthen with increasing Rayleigh number, and the oscillatory state may be developed in the local region if the Rayleigh number is larger than a critical value. It implies that the Marangoni number is not the only parameter of dynamical similarity, and the oscillatory state depends usually on several parameters such as $\mathrm{Ga}$, $\mathrm{Pr}, \mathrm{Bo}$ and others. Therefore, it is reasonable to understand that the critical Marangoni number may be different for the thermocapillary convection in different environments. The calculations are presented elsewhere in detail[18].

\section{CRITICAL MARANGONI NUMBER}

Let us now estimate the value of the critical Marangoni number. According to eqn (1), the Marangoni number may be rewritten as

$$
\mathrm{Ma}=\frac{\alpha \mathrm{Ra}}{\mathrm{Bo}},
$$

where the Bond number is $\mathrm{Bo}=\rho q \beta L^{2} /|\mathrm{d} \sigma / \mathrm{d} T|$; it depends only on the properties of liquid and the gravity environment, $\alpha=\Delta T / \delta T$ is the ratio of applied temperature difference and reversed temperature difference. Equation (2) shows that the critical Marangoni number is proportional to $(\mathrm{Ra})_{\mathrm{c}}$ and inversely proportional to Bo. The value of Bo are, respectively, $0.1,10^{-2}$ and $10^{-3}$ for the experiments on the ground, in rocket flight and on-board the Space Shuttle. Therefore, the critical Marangoni number of thermocapillary convection in a liquid bridge will be one order of magnitude larger in the Space Shuttle than in the rocket, than on the ground; as suggested from the present experiments.

In the problem of Bénard convection, the dynamical similarity parameter is Ra. The Bénard cells are developed by the instability of buoyant force when the value of $\mathrm{Ra}$ reaches 1700 , and the temperature oscillation, and furthermore the turbulent flow, may be developed as $\mathrm{Ra}$ increases further. Therefore, the physical processes defined by $(R a)_{c}$ is not quite the same as one defined by $(\mathrm{Ma})_{c}$. In the detailed calculations, we adopt $\alpha=1.5$ and $(\mathrm{Ra})_{c}=3000$. Silicon oil is used as an experimental medium of which the gradient of surface tension is $\mathrm{d} \sigma / \mathrm{d} T=-0.1 \mathrm{dyn} / \mathrm{cm}$ $\mathrm{K}$. The calculated values of $\mathrm{Bo}$ and $(\mathrm{Ma})_{c}$ are given in Table 1 for different experimental environments. It shows that the calculated value of $(\mathrm{Ma})_{c}$ agrees with the experimental results.

According to the previous analysis based on the physical process a qualitative picture could be obtained. In the case of small $\mathrm{Ma}$, the process of thermal conduction is dominant, and the temperature distribution in the gap of the liquid bridge is nearly linear. The convection speed-up by surface tension gradient as increasing $\mathrm{Ma}$, and the heat is transferred by convection. The thermal boundary layers near the upper and lower rods become thinner and thinner, and then the temperature gradient starts turning in the opposite direction in the local region. In this case. the effect of buoyant forces is gradually important, and the oscillation may finally be developed. This picture implies that the physical mechanisms are similar for both the experiments on ground and in space, although they have different values of $(\mathrm{Ma})$. The space experiment results of the microgravity environment may be simulated by ground laboratory experiments with small Bond number. According to the smaller Bond number in Space than on the ground, the critical Marangoni number is relatively large and the appearance of the oscillatory state is delayed in space. This is favourable for space materials process.

In the previous analysis of thermodynamical process, we assume to use only $R \mathbf{a}$ as the dynamical similarity parameter. This is over-simplified for such a problem as thermocapillary convection in liquid bridge. However, this simple analysis shows a clear physical picture; a better analysis may be obtained after more detailed experimental and theoretical research.

Table 1. The values of Bo and $(\mathrm{Ma})_{\mathrm{c}}$ for silicon oil

\begin{tabular}{lccc}
\hline $\begin{array}{c}\text { Experimental } \\
\text { environment }\end{array}$ & Ground & Rocket & Space Shuttle \\
\hline Terms & 1 & $10^{-3}$ & $10^{-5}$ \\
Gravity level $\left(g / g_{0}\right)$ & 2 & 20 & 60 \\
Height $L(\mathrm{~mm})$ & 0.376 & 0.038 & 0.0034 \\
Bond number Bo & $1.2 \times 10^{4}$ & $1.2 \times 10^{5}$ & $1.3 \times 10^{6}$ \\
Calculated $(\mathrm{Ma})_{\mathrm{c}}$ & $\sim 10^{4}$ & $(1.0-2.0) \times 10^{5}$ & $>4 \times 10^{5}$ \\
Experimental $(\mathrm{Ma})_{\mathrm{c}}$ & & & \\
\hline
\end{tabular}




\section{REFERENCES}

1. C. G. M. Marangoni, Ann. Phys. 143, 337 (1871).

2. D. B. R. Kenning, Appl. Mech. Rev. 21, 1101 (1968)

3. V. G. Levich and V. S. Krylov, Ann. Rev. Fluid Mech. 1, 293 (1969)

4. J. R. A. Pearson, J. Fluid Mech. 4, 489 (1958).

5. L. E. Scriven and C. V. Sternling, J. Fluid Mech. 19, 320 (1964).

6. C. S. Yeh, Phys. Fluid 11, 477 (1968).

7. S. Ostrach, Ann. Rev. Fluid Mech. 14, 313 (1982)

8. C. E. Chang and W. R. Wilcox, J. Crystal Growth 28, 8 (1975).

9. S. Ostrach, Physicochemical Hydrodynamics, . Vol. 2, p. 571, Advanced Publications, New York (1977).
10. S. Ostrach and A. Predhan, AIAA J. 16, 419 (1978).

11. L. G. Napolitano, 2nd Levich Conference, Washington. Unpublished.

12. C.-H. Chun and W. Wuest, Acta Astronautica 5, 681 (1978).

13. C.-H. Chun and W. Wuest, Acta Astronautica 6, 1073 (1979).

14. D. Schwabel and A. Scharmann, J. Crystal Growth 46, 125 (1979).

15. F. Preisser et al., J. Fluid Mech. 126, 545 (1983)

16. Y. Kamotani and S. Ostrach, Paper AIAA-86-0200 (1986).

17. R. Monti, Acta Astronautica 15, 557 (1987).

18. W.-R. Hu and Z.-M. Tang, Submitted to Scientia Sinica. 